\title{
Precision and control
}

Gene therapy promises many new ways to combat disease. The best strategy depends on the disease, but two important goals are that the genetic change is precise, and that gene expression is controlled according to physiological needs. Two new studies report progress towards both of these aims.

For inherited diseases, precise correction of the causative mutation could be achieved by homologous recombination. The best opportunities for this are afforded by diseases of the haematopoietic system. Haematopoietic stem cells (HSCs) can be harvested from a patient, modified ex vivo, and transfused back into the patient. Hatada et al. isolated bone marrow cells from Hprt-deficient mice (a model for LeschNyhan syndrome), and corrected the Hprt gene by homologous recombination at a frequency similar to that achieved in embryonic stem cells. Although the corrected cells are haematopoietic progenitor cells - not HSCs - the results will encourage further efforts to modify HSCs by homologous recombination.

In the case of a more complex multifactorial disease such as diabetes, gene therapy can offer a different approach to disease treatment. The key problem in type I diabetes sufferers is a lack of insulin. The production of insulin is regulated in response to glucose, so combatting diabetes using gene therapy requires an expression system that is regulable in vivo. Lee and colleagues created an insulin expression vector containing a glucose-regulable promoter. The vector corrected insulin deficiency in rat and mouse models for type I diabetes for at least several months after vector transduction and reproduced the physiological coordination of insulin levels with those of glucose. There are some important questions to consider before a similar approach can be contemplated in humans but, as in the study by Hatada et al., Lee et al. have shown how gene-therapy technology can be improved to bring precision and control a little closer.

Mark Patterson

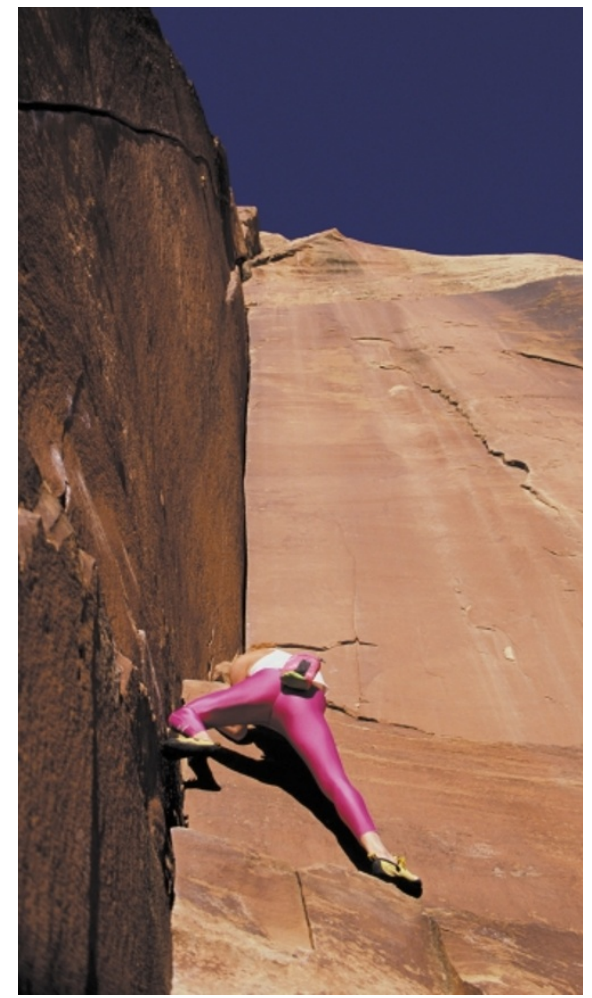

(6) References and links ORIGINAL RESEARCH PAPERS Hatada, S. et al. Gene correction in hematopoietic progenitor cells by homologous recombination. Proc. Natl Acad. Sci. USA 97, 13807-13811 (2000) | Lee, H. C. et al. Remission in models of type 1 diabetes by gene therapy using a single-chain insulin analogue. Nature 408, 483-488 (2000) FURTHER READING Olefsky J. M. Gene therapy for rats and mice. Nature 408, 420-421 (2000)

\section{GENE EXPRESSION}

\section{Escaping silence}

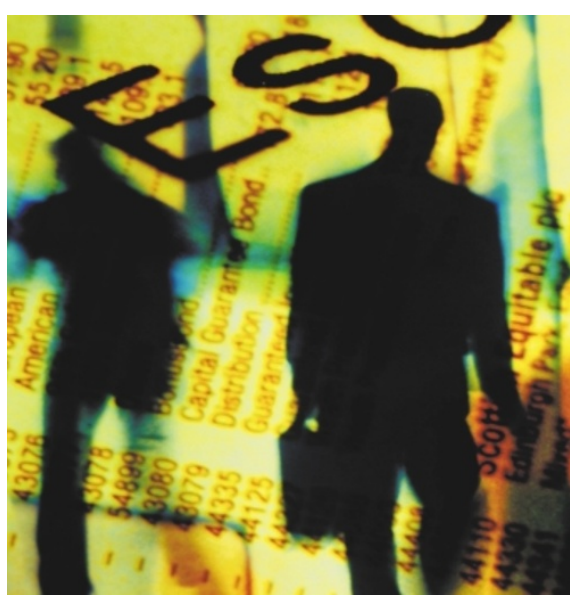

What silences genes - promoter methylation, location within heterochromatin or late cellcycle replication? The answer seems to lie not with any one of these events but with all of them (and maybe more). A stable genesilencing event that requires the (mostly unknown) interactions of many genesilencing processes is $\mathrm{X}$ inactivation. To tease apart such interactions, Hansen et al. turned to cells from individuals with defective methylation to investigate the relationship between promoter methylation, replication timing and gene silencing.

The authors used fibroblasts and lymphoblasts from patients with ICF (immunodeficiency, centromeric instability and facial anomalies) syndrome - a human disorder caused by mutations in DNMT3B, which encodes the 'de novo' DNA methyltransferase $3 \beta$. These mutations do not cause genome-wide methylation defects but hypomethylation at particular heterochromatic regions, such as at pericentromeric satellite DNA and the inactive $\mathrm{X}$ chromosome

From their studies, Hansen et al. found that in both primary and transformed ICF cells, normally hypermethylated $\mathrm{CpG}$ islands at the promoters of several inactive $\mathrm{X}$ genes were hypomethylated. However, this hypomethylation did not lead to a wholesale escape from transcriptional silencing - only two of the eleven genes that were studied, G6PD and SYBL1, showed biallelic expression in ICF cells. The authors then found that an advance in the replication timing of these genes correlated with their escape from inactivation - in ICF fibroblasts, for example, G6PD and SYBL1 replicate earlier in the cell cycle than they do in normal cells.

But the relationships between silencing, replication timing and methylation are far from being this simple — several hypomethylated, inactive $\mathrm{X}$ genes from ICF cells also replicate earlier than normal but remain silent, perhaps because their replication is still not as early as that of active $\mathrm{X}$ alleles. Also, hypomethylated promoter chromatin is sensitive to nuclease digestion at both reactivated and silent genes on the inactive $\mathrm{X}$ from ICF cells. These results indicate that advanced replication can occur without transcription, and that it might therefore be a cause rather than a consequence of escape from inactivation. Late replication on the inactive $\mathrm{X}$ might be maintained or established by DNMT3B; in its absence, other factors might assume this role with less fidelity.

Much remains to be learnt about how higher-order genomic organization influences nuclear compartmentalization and replication timing to control gene expression. ICF cells look set to provide a handy tool for such investigations and may provide new insights into how deregulated gene expression contributes to the ICF phenotype.

Jane Alfred

6) References and links

ORIGINAL RESEARCH PAPER Hansen, R. S. et al. Escape from gene silencing in ICF syndrome: evidence for advanced replication time as a major determinant. Hum. Mol. Genet. 9 2575-2587 (2000)

FURTHER READING Jones, K. A. \& Kadonaga, J. T.

Exploring the transcription-chromatin interface. Genes Dev.

14, 1992-1996 (2000)

WEB SITES Stanley Gartler's and Scott Hansen's labs 